\title{
Introduction: Special Issue on Innovative Medicine and Research: Ethical, Legal and Regulatory Issues
}

The process of translating biomedical advancements in biology, biotechnology and genomics into new therapies and products has become increasingly complex. Today genetics, pharmacogenetics, cell therapies, imaging, information and communications technology (ICT) applications represent the most advanced tools incorporated in the drug discovery and research and development process used for producing the largest part of the innovative medicines already available on the market or under development. More recently, Health Technology Assessment (HTA) has demonstrated to be one important part of the medicines' lifecycle by providing scientific evidence-based decisions on the pricing and reimbursement of health technologies. Furthermore, innovative medicine and research are not only concerned with new technologies and products, but also about people, behaviour and the need for change in the mindset of all stakeholders including patients to enable innovation to thrive.

It is in this context that guidance and regulation have to be continuously adapted and updated to guarantee that scientific discoveries are translated into innovative clinical solutions for unmet needs that reach patients. Therefore, innovation in medicine development and research should not only be assessed from a scientific perspective, but also in a wider context whereby clinical benefits, economic/health-outcome improvements, ethical, legal and regulatory issues are considered while guarantying the respect of fundamental and patients' rights.

To ensure the alignment of legal and regulatory issues with scientific and clinical objectives, scientists, regulatory and commercial interested parties need to cooperate. Moreover, professionals and patients should have access to the decisional and regulatory processes; academia and researchers should be fully involved, so as to meet social and ethical expectations.

The Fondazione per la Ricerca Farmacologica Gianni Benzi Onlus (Gianni Benzi Foundation for pharmaceutical research) operates by integrating in its mission and scope Professor Benzi's forward-thinking idea that the European pharmaceutical system is key to both scientific development and economic growth, taking ethical and social impacts into account. Thus, the Foundation promotes annual FGB-Foresight Training Courses (FTC), short international 
courses aimed at training highly-specialised professional profiles in the different fields of regulatory sciences. They also represent a relevant occasion to disseminate good practices, to promote pharmacology, health science, ethics and regulatory research and innovation among the main stakeholders (regulatory experts from industries, patients, healthcare professionals, no profit organisations and researchers from academia).

Within its statutory missions and taking this multidisciplinary approach into account, the Gianni Benzi Foundation for Pharmaceutical Research organised, under the coordination of Annagrazia Altavilla and Prof. Adriana Ceci, and in collaboration with the Espace Ethique PACA-Corse, a workshop as a special session of its Foresight Training course, held during the 7th Conference of the European Association of Health Law (EAHL) (Toulouse, France 25-27 September 2019) under the patronage of the Council of Europe. Eminent experts from nine European countries participated in this workshop.

Their contributions are included in this special issue of the European Journal of Health Law devoted to 'Innovative Medicine and Research: Ethical, Legal and Regulatory Issues'.

As underlined, to face innovation in healthcare, a multidisciplinary approach is of paramount importance to better understand the scientific, legal, regulatory and ethical challenges. In this perspective, we strongly believed that it was necessary to also include contributions from non-legal professionals in this special issue, in order to have a different and complementary point of view that would support academics and legal professionals in appraising the scientific and societal issues at stake. It could also help to understand how legal procedures and current regulatory framework should be adapted to the scientific progress. In this issue, the following topics are addressed: innovative medicine and research; access to healthcare and innovation; empowerment and patients' rights in innovative healthcare.

In particular, with reference to research, Jean Herveg, Head of the LIS Department of the Research Centre on Information, Law and Society (CRIDS) of the University of Namur and Annagrazia Altavilla provide an overview of key elements that should be taken into account when considering new ways to organise access to personal data for scientific research in the perspective of developing innovative medicines. Introducing different models of data sharing (such as the theory of the essential facilities, the EU Regulation on the free flow of non-personal data in the European Union, the EU Directive on open data and the re-use of public sector information, and the EU General Data Protection Regulation (GDPR) rules on accessing personal data for scientific research) Jean Herveg points out the need for clarification and further investigations of each of them, with the aim to clarify their applicability and 
efficacy to foster scientific research while safeguarding fundamental rights. Annagrazia Altavilla also underlines the need to focus on ethics and patients' rights issues in the evaluation of the data access models.

In the paediatric perspective, Annagrazia Altavilla and other experts from TEDDY and EPTRI, ${ }^{1}$ after having clarified the concept of translational research in the perspective of the establishment of a European Paediatric Research Infrastructure (RI), identify and point out the ethical, legal and regulatory issues particularly relevant in a children rights' perspective. Given the great number of unsolved and uncovered ethical and legal issues related to paediatric translational research, Annagrazia Altavilla highlights how crucial the role of paediatric networks and European research infrastructure is in identifying elements to be taken into account by different stakeholders involved in paediatric research. Conceiving a new model of governance of a paediatric translational research infrastructure, a multidisciplinary 'common service' should be set up to provide the correct strategy to address the uncovered issues while ensuring compliance with relevant rules. Such a model could help to come to coherent decisions, based on common European values, and harmonised practices in accordance with ethical and legal norms, always considering the best interests of the child.

With the aim to map and describe the landscape of the protection of genetic digital sequence information (DSI) and the application of intellectual property rights to it, Claudia Seitz, Lecturer and Visiting Professor at Basel and Ghent Universities, sheds light on some burning questions to reflect the current and ongoing discussion. She stresses how increasingly complex challenges linked to the regulation of DSI and its technological use are in a rapidly changing scientific environment. Outlining its legal, policy and scientific implications, she calls for an open discussion on the question whether provisions of the Nagoya Protocol should be amended to address the regulation of DSI, and to clarify the questions regarding the applicability of the current ABS system. ${ }^{2}$ The answer to these questions will be of fundamental importance for the international sciences community in the context of a free and open access to sequence information and will also have an impact for new products (such as vaccines) to protect the human health.

Fedele Bonifazi, President of the Gianni Benzi Foundation, and other experts from the Foundation, provide an overview of practical application of machine

1 TEDDY - European Network for Paediatric Research and EPTRI - the European Paediatric Translational Research Infrastructure.

2 Nagoya Protocol on Access to Genetic Resources and the Fair and Equitable Sharing of Benefits Arising from Their Utilization (ABS). 
learning (ML) in an innovative healthcare context. With a descriptive/state of the art and technical approach, they describe the challenges linked to the ML systems and explain how sustainable the regulatory system in relation to the ML innovation is. The challenges are numerous: regulatory issues linked to standardisation and safety; ethical and legal issues linked e.g., to the question of liability; bias and questions about the fiduciary relationship between patients and ML systems. Issues of this kind should be addressed without delay. Interdisciplinary validation of AI systems is therefore needed to ensure their reliability and their integration through adequate legal and regulatory implementation, programming approaches, and task force work, evaluating societal impact and acceptance.

Vincenzo Salvatore, former Head of the Legal Service at the European Medicines Agency and Professor at the Insubria University in Italy, analyses the strengths and weaknesses of Advanced Therapies Medicinal Products (ATMPs) EU Regulation. Exploring possible ways to foster the development of ATMPs in Europe and expand patients' access to these new treatments, he stresses the need of more transparency and harmonisation of criteria for hospital exemptions across the EU and for more guidance from Member States. In addition, he underlines the need for a closer and more fruitful cooperation and interaction between regulatory authorities, industry, universities, hospitals and research institutions. Strengthened mechanism of sharing information at an early stage of research and development of ATMPs, in fact, would allow all the relevant key players to support each other and to become aware of the pipeline and of the need to comply with regulatory requirements.

When healthcare innovations are proven safe and ready for application, decision makers sometimes have to face difficult assessments to implement access policies. Different methods and access policies are used throughout Europe to achieve equitable access to publicly-funded healthcare of appropriate quality.

In terms of access to healthcare and innovation, after illustrating current challenges with the example of hepatitis B, Verena Stühlinger, senior scientist at UMIT University and Vice-President of the EAHL, provides an overview of different access policies and tools for decision support, which aim to guarantee equitable access to healthcare in the EU. She critically discusses reasons for and effects of the current fragmentation of HTA-strategies and examines latest developments at EU-level. In this scenario, she underlines that HTA plays a crucial role at EU-level, forming an evidence-based, transparent basis for decision making through joint assessments and concludes highlighting that, in a highly fragmented decision-making system, great efforts have to be taken in order to bring all stakeholders together and inspire them for more cooperation. 
From a patient' empowerment perspective, Eloise Gennet, researcher at the University of Toulouse -Bioethics Team, Inserm Unit 1027, introduces a human rights-based approach to the concept of 'health vulnerability' in the context of rare diseases. In order to guarantee a significant improvement in patients' access to orphan medicines, she suggests to link the promotion of the health of orphan disease patients to the growing concept of vulnerability and, specifically, to a collective dimension of health vulnerability. Using this normative concept will permit embedding the development of orphan drugs and the promotion of patients' access to quality orphan medicines within the perspective of the protection of fundamental rights, as opposed to a simple incentivising regime (actually provided by the Regulation on orphan medicinal products), whose implementation is highly dependent on an economic and political context. She also underlines that the human-rights based approach would be even more valuable as it would simultaneously draw attention on privacy aspects for orphan disease patients, especially regarding increased risks stemming from the processing of highly sensitive health data.

Always in the field of rare diseases, François Houyez, Information and Access to Therapies Director and Eurordis Health Policy Advisor, after having elucidated the specificity of orphan medicinal products price, provides an overview of some important actions carried out by patients and their organisations. Underling the collective need to reduce the cost of innovation, he analyses specific actions to be undertaken to guarantee access to innovative drugs especially for rare diseases. He also highlights the need to promote more initiatives in Europe in order to face the problem of the high cost of medicines taking also into account 'the right of everyone to benefit from medical treatment', as well as the principle of the 'equality of treatment' of European citizens. Public debates aimed at increasing awareness on this issue should be encouraged as well.

Scientific and technological developments require a complementary approach to legal instruments; they call for new and adequate models of governance in the biomedical field to be developed according to human rights and societal values. From this perspective, Siobhan O'Sullivan, Chair of the Council of Europe (COE) DH-BIO Drafting Group on the Strategic Action Plan for 2020-2025, and Vice-chair of the European Group on Ethics in Science and New Technologies (GEE), analyses the European models of governance that guide innovation in the healthcare sphere. There is an increasing realisation that legal instruments need to be supplemented with public discourse to help inform questions about how we want to shape our lives and societies, who makes the necessary decisions, and through what fora and institutions. That is why, from the earliest stages of innovation, governance has to include 
elements such as the respect of human rights and the rule of law, transparent and accountable processes and institutions and effective participation. In order to guarantee that the directions of innovation and the ethical challenges raised by technological developments are extensively deliberated, democratic and legitimate governance should go hand in hand with a process of societal deliberation, involving expert, political, and public opinions.

In the same perspective, Laurence Lwoff, Head of the Head of the Bioethics Unit of the Council of Europe, outlines the main issues considered by the Committee on Bioethics of the COE. In particular, she points out that consideration is required as to whether existing human rights provisions are still fit for purpose or whether there is a need to re-examine, clarify or re-enforce them or even a need to identify new human rights and protective measures. She confirms that public discourse/dialogue is an essential component. The vigilance and the responsible involvement of all actors of our societies are also required. Finally, she gives a view to the new Strategic Action Plan on Human Rights and Technologies in Biomedicine (2020-2025) developed by the Committee on Bioethics of the Council of Europe.

The Strategic Action Plan, based on human rights, is articulated around four main pillars (governance, equity of access to healthcare, mental and physical integrity and communication and cooperation) under which strategic objectives are defined. Among other objectives, we should stress the strengthening of children's participation in the decision-making process on matters regarding their health, as well as the safeguarding of children's rights in relation to medical practices that may have future and/or long-term implications for them.

Despite the specificities of the topics analysed, all of the authors agree that innovation in medicine and emerging technologies require adequate models of governance and coordinate actions aimed at promoting responsible research, technology applications and access policies based on human rights as well as increased citizen participation and reflexivity. Such participation, a key element of good governance, will contribute to ensure that human rights considerations are observed. This will maximise the likelihood that from the outset developments are beneficial and oriented towards protecting ethical and societal values. Thus, all the authors in this issue call for more transparency, more collaboration among the main stakeholders (academics, researchers, health/legal professionals, industry, institutions, patients, children...), as well as further research and initiatives to increase the awareness of the public and promote public debates. May this workshop and its collaborative work be the first of further common initiatives promoting discussion on ethical, legal and societal issues within a human-rights approach. 
Many thanks to the Gianni Benzi Foundation for Pharmaceutical Research for supporting this event and collaborative work, and particularly to Professor Adriana Ceci for her strong and tireless engagement in always promoting innovative initiatives and projects, as well as to Anne-Marie Duguet for the great and successful organisation of the 7th EAHL Conference held in Toulouse, and to all the authors for the fruitful exchanges and their contributions on these challenging topics.

\section{Annagrazia Altavilla ${ }^{3}$}

Guest Editor

3 Fondazione per la Ricerca Farmacologica Gianni Benzi Onlus, Italy; Espace Éthique Méditerranéen/PACA-Corse, AP-HM, France; TEDDY European Network for Paediatric Research - Chair; EAHL, Member of the Board of Directors - French representative, annagrazia.altavilla@univ-amu.fr. 